\title{
Dark energy from a quintessence (phantom) field rolling near potential minimum (maximum)
}

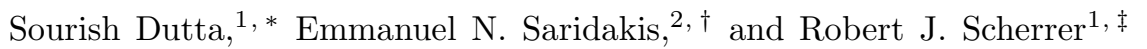 \\ ${ }^{1}$ Department of Physics and Astronomy, Vanderbilt University, Nashville, TN 37235 \\ ${ }^{2}$ Department of Physics, University of Athens, GR-15771 Athens, Greece
}

\begin{abstract}
We examine dark energy models in which a quintessence or a phantom field, $\phi$, rolls near the vicinity of a local minimum or maximum, respectively, of its potential $V(\phi)$. Under the approximation that $(1 / V)(d V / d \phi) \ll 1$, [although $(1 / V)\left(d^{2} V / d \phi^{2}\right)$ can be large], we derive a general expression for the equation of state parameter $w$ as a function of the scale factor for these models. The dynamics of the field depends on the value of $(1 / V)\left(d^{2} V / d \phi^{2}\right)$ near the extremum, which describes the potential curvature. For quintessence models, when $(1 / V)\left(d^{2} V / d \phi^{2}\right)<3 / 4$ at the potential minimum, the equation of state parameter $w(a)$ evolves monotonically, while for $(1 / V)\left(d^{2} V / d \phi^{2}\right)>3 / 4, w(a)$ has oscillatory behavior. For phantom fields, the dividing line between these two types of behavior is at $(1 / V)\left(d^{2} V / d \phi^{2}\right)=-3 / 4$. Our analytical expressions agree within $1 \%$ with the exact (numericallyderived) behavior, for all of the particular cases examined, for both quintessence and phantom fields. We present observational constraints on these models.
\end{abstract}

\section{INTRODUCTION}

It has been known for over a decade [1, 2] that at least $70 \%$ of the energy density in the universe is in the form of an exotic, negative-pressure component, called dark energy (see Ref. [3] for a recent review). The equation of state of this dark component, defined as the ratio of its pressure to its density:

$$
w=p_{\mathrm{DE}} / \rho_{\mathrm{DE}},
$$

is observationally constrained to be close to -1 . In particular, constraints on constant $w$ lead typically to $w=$ -1 with $\pm 10 \%$ accuracy [4, [5, 6]. However, a variety of cosmological paradigms, based on scalar fields, attribute a dynamical nature to dark energy, leading to a timevarying $w$. The class of models in which the scalar field is canonical is dubbed quintessence $7,8,8,9,10,11,12]$ and has been extensively studied in the literature. An alternative approach is phantom dark energy, a component for which $w<-1$, which can be realized by using a scalar field with a negative kinetic term in its Lagrangian, as first proposed by Caldwell [13]. Such models have wellknown problems [14, 15, 16, 17], but nevertheless have also been widely studied as potential dark energy candidates $13,18,19,20,21,22,23,24,25,26,27,28,29$. Finally, since both quintessence and phantom models cannot lead to a dark energy equation-of-state parameter that crosses -1 during cosmological evolution (which might be the possibility according to observations), the simultaneous consideration of both models in a joint scenario, named quintom, has recently gained significant attention [30, 31, 32, 33, 34, 35].

\footnotetext{
*Electronic address: sourish.d@gmail.com

${ }^{\dagger}$ Electronic address: msaridak@phys.uoa.gr

‡Electronic address: robert.scherrer@vanderbilt.edu
}

Given the considerable freedom that exists in choosing the potential function $V(\phi)$ of the scalar field, it is useful to develop general expressions for the evolution of $w$ which cover a wide range of models. A general result of this type was derived in [36] for a class of quintessence models in which $w$ is assumed to be always close to -1 and the potential is nearly flat. The flatness of the potential is characterized by two "slow roll conditions":

$$
\left(\frac{1}{V} \frac{d V}{d \phi}\right)^{2} \ll 1
$$

and

$$
\frac{1}{V} \frac{d^{2} V}{d \phi^{2}} \ll 1
$$

For these models, it was shown in [36] that the evolution of $w$ is described by a unique expression involving only the present values of $\Omega_{\phi}$ and $w$. In [37] this result was extended to phantom models satisfying (2)-(3), and the corresponding $w$ dependence was shown to be described by the same expression with an overall sign change. Finally, the extension to quintom scenario under (2)-(3) was performed in [38], where a universal expression for $w$ was also extracted, allowing for crossing of $w=-1$.

While the slow-roll conditions (2) and (3) are sufficient to give $w \simeq-1$ today, they are not necessary. Classes of models characterized by the validity of (2) alone, i.e., without (3), were considered in 39], corresponding in particular to a quintessence field in the vicinity of a local maximum of its potential. In this case, there is an extra degree of freedom, namely the value of $(1 / V)\left(d^{2} V / d \phi^{2}\right)$, which describes the curvature of the potential in the vicinity of the maximum. As a result, instead of a single solution for the evolution of $w$ one obtains a family of solutions that depend on the present-day values of $\Omega_{\phi}$ and $w$ and the value of $(1 / V)\left(d^{2} V / d \phi^{2}\right)$ at the maximum of the potential. As expected, this family of solutions includes the slow-roll solution of [36] as a special case in 
the limit where $(1 / V)\left(d^{2} V / d \phi^{2}\right) \rightarrow 0$. In [40], a similar result was derived for a phantom field evolving near a minimum of its potential. It was found that a unique family of solutions, very similar to the one derived in [39], can be used to approximate the behavior of $w$ in these models.

More recently, Chiba [41] showed that while conditions (2) and (3) are sufficient to allow the quintessence version of the slow-roll conditions to be applied, they are not necessary. Chiba extended the methodology of Ref. [39] to provide a more general set of conditions on the potential, dropping the assumption that the field is close to a local maximum in the potential, while still assuming that $w$ is close to -1 throughout the evolution. Interestingly, the expression derived under these more general conditions coincides exactly with the expression derived in [39], indicating the generality of this result.

In this work we extend the techniques developed in [39] to the "opposite" of the case considered there. In Ref. [39], the quintessence field was considered to roll near a local maximum in the potential, so that $V^{\prime \prime}>0$. In this paper, we consider the case for which $V^{\prime \prime}<0$. This corresponds to a canonical scalar field rolling close to a minimum of its potential. For completeness, we also examine the corresponding phantom model, for which the phantom field rolls near a maximum of its potential. That is, while in [39] and [40] the field rolls away from an unstable potential-extremum, in the present work the field rolls towards a stable extremum. (Recall that phantom fields roll away from local minima and are attracted by local maxima). As we will see, this investigation yields a more complicated dynamics for the scalar field, which can include oscillations in addition to slow roll behavior. For simplicity, we assume that condition (2) is satisfied, and that the field evolves near an extremum in the potential, rather than making the more general assumptions of Ref. [41]. The result can be generalized, in a straighforward way, following the development of Ref. [41]. We note further than our main result is mentioned in Ref. [41], although it is not investigated in detail there.

The plan of our paper is as follows: In section II we construct the model of scalar field evolution near a stable potential extremum, and we derive the expression for $w(a)$. In section III we apply our formula to various potentials, comparing the results to the exact evolution arising by numerical integration. We then constrain this general family of models with SNIa observations. Our results are summarized in section [V].

\section{FIELD EVOLUTION NEAR A STABLE POTENTIAL-EXTREMUM}

We are interested in models where the scalar field evolves near a stable potential-extremum, and thus we consider a minimally coupled scalar field $\phi$ in a potential $V(\phi)$, where the field $\phi$ can be either a canonical or a phantom one. In the following we introduce the usual $\varepsilon$ - parameter, acquiring the value +1 for the canonical case, and -1 for the phantom one. Doing so we can present our expressions in a general way.

The Euler-Lagrange equation of motion of the field reads:

$$
\ddot{\phi}+3 H \dot{\phi}+\varepsilon \frac{d V}{d \phi}=0,
$$

where $a$ is the scale factor and $H \equiv \dot{a} / a$ is the expansion rate. Dots denote derivatives with respect to time and primes denote derivatives with respect to the field $\phi$. In a flat universe, the expansion rate is linked to the total density $\rho_{\mathrm{T}}$ via the first Friedman equation (in units where $8 \pi G=1)$ as

$$
H^{2}=\rho_{\mathrm{T}} / 3
$$

Additionally, the evolution of the scale factor is given by:

$$
\frac{\ddot{a}}{a}=-\frac{1}{6}\left(\rho_{\mathrm{T}}+p_{\mathrm{T}}\right),
$$

where $p_{\mathrm{T}}$ is the total pressure.

Following [39] we perform the transformation

$$
\phi(t)=u(t) / a(t)^{3 / 2},
$$

and therefore Eq. (4) becomes

$$
\ddot{u}+\frac{3}{4} p_{\mathrm{T}} u+\varepsilon a^{3 / 2} V^{\prime}\left(u / a^{3 / 2}\right)=0 .
$$

We consider a universe consisting of pressureless matter and a scalar field, where the scalar field plays the role of the dark energy. In order to realistically mimic the observed dark energy, the scalar field must have $w$ close to -1 and its energy density must be roughly constant. The total pressure $p_{\mathrm{T}}$ is then simply given by $p_{\mathrm{T}} \approx-\rho_{\phi 0}$, where $\rho_{\phi 0}$ is the present day density of the dark energy. (In what follows, a subscript 0 always indicates a present day value). Under this approximation, Eq. (8) becomes:

$$
\ddot{u}-\frac{3}{4} \rho_{\phi 0} u+\varepsilon a^{3 / 2} V^{\prime}\left(u / a^{3 / 2}\right)=0 .
$$

Since we are interested in applying Eq. (9) to a scalar field evolving near a local potential-extremum at $\phi_{*}$, for any $\phi$ close $\phi_{*}$ we use the expansion:

$$
V(\phi)=V\left(\phi_{*}\right)+(1 / 2) V^{\prime \prime}\left(\phi_{*}\right)\left(\phi-\phi_{*}\right)^{2}+O\left(\left(\phi-\phi_{*}\right)^{3}\right) .
$$

Substituting the above relation into Eq. (9), and imposing $V\left(\phi_{*}\right)=\rho_{\phi 0}$ we obtain the following differential equation for the field evolution:

$$
\ddot{u}-\left[(3 / 4) V\left(\phi_{*}\right)-\varepsilon V^{\prime \prime}\left(\phi_{*}\right)\right] u=0 .
$$

This is essentially the same equation derived previously in Ref. [39].

In this work we examine cosmological evolution near a stable potential-extremum, that is either a canonical 
field $(\varepsilon>0)$ near a local minimum $\left(V^{\prime \prime}\left(\phi_{*}\right)>0\right)$, or a phantom field $(\varepsilon<0)$ near a local maximum $\left(V^{\prime \prime}\left(\phi_{*}\right)<\right.$ $0)$. Thus, in both cases of interest $\varepsilon V^{\prime \prime}\left(\phi_{*}\right)>0$ and therefore the following analysis can be performed in a unified way.

Defining

$$
k \equiv \sqrt{(3 / 4) V\left(\phi_{*}\right)-\varepsilon V^{\prime \prime}\left(\phi_{*}\right)},
$$

we obtain the general solution of Eq. (11) as

$$
u=A \sinh (k t)+B \cosh (k t),
$$

where $A$ and $B$ are arbitrary constants. Note that this solution holds for $k$ being either real or imaginary, where in the latter case the hyperbolic functions are straightforwardly replaced by trigonometric ones. Hence, we can simply use the earlier results of Ref. [39].

Defining

$$
t_{\Lambda} \equiv 2 / \sqrt{3 \rho_{\phi 0}}=2 / \sqrt{3 V\left(\phi_{*}\right)}
$$

and taking $\phi(t=0)=\phi_{i}$, we obtain

$$
\phi=\frac{\phi_{i}}{k t_{\Lambda}} \frac{\sinh (k t)}{\sinh \left(t / t_{\Lambda}\right)}
$$

and the equation of state parameter, $w(a)$, is given by (see Ref. [39] for the details):

$$
1+w(a)=\left(1+w_{0}\right) a^{-3} \frac{\left[\sqrt{\Omega_{\phi 0}} k t_{\Lambda} \cosh [k t(a)]-\sqrt{\left(1-\Omega_{\phi 0}\right) a^{-3}+\Omega_{\phi 0}} \sinh [k t(a)]\right]^{2}}{\left[\sqrt{\Omega_{\phi 0}} k t_{\Lambda} \cosh \left(k t_{0}\right)-\sinh \left(k t_{0}\right)\right]^{2}},
$$

where $w_{0}$ is the present-day value of $w(a)$, and $t(a)$ and $t_{0}$ can be derived from:

$$
t(a)=t_{\Lambda} \sinh ^{-1} \sqrt{\left(\frac{\Omega_{\phi 0} a^{3}}{1-\Omega_{\phi 0}}\right)}
$$

and

$$
t_{0}=t_{\Lambda} \tanh ^{-1}\left(\sqrt{\Omega_{\phi 0}}\right)
$$

Note that in (16) the $\varepsilon$-parameter has been simplified in favor of $w_{0}$ which is obviously smaller than -1 for phantom while it is larger than -1 for the quintessence case.

Following Ref. [39], we now introduce the constant $K \equiv k t_{\Lambda}$. In terms of the potential, $K$ can be written as

$$
K=\sqrt{1-\varepsilon \frac{4 V^{\prime \prime}\left(\phi_{*}\right)}{3 V\left(\phi_{*}\right)}}
$$

In terms of $K$ we can finally express the evolution of $w$ in the following form:

$$
1+w(a)=\left(1+w_{0}\right) a^{3(K-1)} \frac{\left[(F(a)+1)^{K}(K-F(a))+(F(a)-1)^{K}(K+F(a))\right]^{2}}{\left[\left(\Omega_{\phi 0}^{-1 / 2}+1\right)^{K}\left(K-\Omega_{\phi 0}^{-1 / 2}\right)+\left(\Omega_{\phi 0}^{-1 / 2}-1\right)^{K}\left(K+\Omega_{\phi 0}^{-1 / 2}\right)\right]^{2}},
$$

where $F(a)$ is given by

$$
F(a)=\sqrt{1+\left(\Omega_{\phi 0}^{-1}-1\right) a^{-3}} .
$$

(Note that $F(a)=1 / \sqrt{\Omega_{\phi}(a)}$, where $\Omega_{\phi}(a)$ is the value of $\Omega_{\phi}$ as a function of redshift, so that $F(a=1)=\Omega_{\phi 0}^{-1 / 2}$.)

Expression (20) is identical in form to the expression for $w(a)$ for a quintessence field near a local maximum 39] or phantom near a local minimum [40] (and see Ref. [41] for the derivation of this expression for more general cases). However, the crucial difference lies in the definition of $K$ (equation 19). In the cases considered in Refs.
[39] and [40], $K$ was always real since $\varepsilon V^{\prime \prime}\left(\phi_{*}\right)<0$ (in the present language). In the case we are considering here, for which $\varepsilon V^{\prime \prime}\left(\phi_{*}\right)>0, K$ can be real or imaginary (see also [41]), corresponding to $\varepsilon V^{\prime \prime} / V<3 / 4$ or $\varepsilon V^{\prime \prime} / V>3 / 4$, respectively. Further, for the special case of $K=0$, equations (15) and (20) are no longer valid; instead we have

$$
\phi=\frac{\phi_{i}}{t_{\Lambda}} \frac{t}{\sinh \left(t / t_{\Lambda}\right)}
$$

and 


$$
1+w(a)=\left(1+w_{0}\right) a^{-3} \frac{\left[\sqrt{\Omega_{\phi 0}}-\sqrt{\left(1-\Omega_{\phi 0}\right) a^{-3}+\Omega_{\phi 0}} \sinh ^{-1}\left(\sqrt{\frac{a^{3} \Omega_{\phi 0}}{1-\Omega_{\phi 0}}}\right)\right]^{2}}{\left[\sqrt{\Omega_{\phi 0}}-\sinh ^{-1}\left(\sqrt{\frac{a^{3} \Omega_{\phi 0}}{1-\Omega_{\phi 0}}}\right)\right]^{2}} .
$$

Now consider the behavior of the scalar field for the three cases of interest, $K^{2}>0, K=0$, and $K^{2}<0$. When $K^{2}>0$, the functional form of equation (20) for quintessence evolution near a potential minimum (or phantom evolution near a local maximum) is identical to the form for the evolution of quintessence near a maximum (or phantom near a minimum) given in Refs. [39, 40], or for the evolution described in Ref. [41]. However, the crucial difference is that in the previouslyconsidered cases, we have $K>1$, while for the case considered here, we have instead $0<K<1$ (the special case $K=1$ is discussed in Ref. [41]). Therefore, although qualitatively the behavior of $w(a)$ is similar to that in Refs. 39, 40, 41, i.e. $w(a)$ decreases monotonically, there are significant quantitative differences.

When $K=0$, we can no longer use equation (20), but equation (23) gives an evolution for $w(a)$ that is qualitatively similar to the $K^{2}>0$ case, i.e., a slow monotonic decrease in $w$.

Finally, when $K^{2}<0, w(a)$ is oscillatory. While equation (20) is formally valid (and gives a real expression for $w(a))$ in this case, the oscillatory behavior becomes more transparent by writing $K=i t_{\Lambda} \gamma$, where $\gamma$ is real, and simplifying Eq. (16) to a more intuitive form (see also Ref. [41]):

$$
1+w(a)=\left(1+w_{0}\right) a^{-3} \frac{\left[\sqrt{\Omega_{\phi 0}} \gamma t_{\Lambda} \cos [\gamma t(a)]-\sqrt{\left(1-\Omega_{\phi 0}\right) a^{-3}+\Omega_{\phi 0}} \sin [\gamma t(a)]\right]^{2}}{\left[\sqrt{\Omega_{\phi 0}} \gamma t_{\Lambda} \cos \left(\gamma t_{0}\right)-\sin \left(\gamma t_{0}\right)\right]^{2}} .
$$

The behavior of $\phi(a)$ for several different values of $K$ is illustrated in Fig. (11). The quantity $K$ can be physically

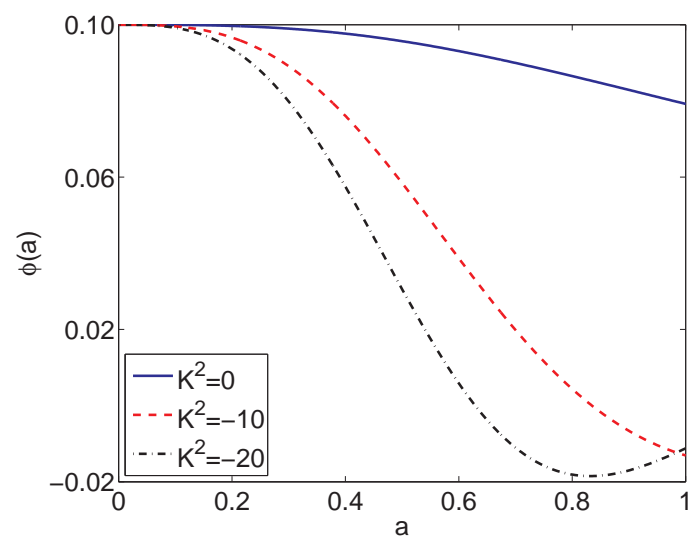

FIG. 1: The evolution of the scalar field $\phi$ as a function of the scale factor $a$, for the indicated values of $K$, as defined in Eq. (19).

interpreted as a measure of the sharpness of the curvature of the potential at its extremum. From the definition of $K$ (Eq. 19) we note that in order for $K$ to be real, $\left|V^{\prime \prime}\right| / V$ is small, implying a "flat" potential. The field evolves in a slow, friction-dominated manner (with the Hubble parameter acting as the friction coefficient according to Eq. 4), asymptotically coming to rest at the potential extremum (minimum for quintessence and maximum for phantom). On the other hand, an imaginary $K$ requires a large $\left|V^{\prime \prime}\right| / V$, implying a sharp curvature of the potential at the extremum, which allows for oscillations since the friction term can be overcome.

It is important to note that we fix the minimum value of the potential to be at the level of the cosmological constant, so the evolution is still potential energy dominated (i.e., $w \simeq-1$ ) as the field oscillates around the extremum. These oscillatory solutions are therefore very different from the ones usually considered in oscillating dark energy models [42, 43, 44, 45, 46, 47], where the potential minimum is fixed at $V=0$, and $w$ oscillates between \pm 1 .

\section{COMPARISON TO EXACT SOLUTIONS}

In this section, we compare our analytic expression for the evolution of $w$ to the numerically computed exact evolution for a few different models. In each case we have a perfect-fluid dark matter and a dark-energy component attributed to a quintessence $(\varepsilon=+1)$ or a phantom $(\varepsilon=$ -1 ) field $\phi$. For the quintessence case, we consider three different potentials which have local minima, and we use 
the corresponding inverted potentials for the phantom case. Our purpose is not to propose these toy models as specific possibilities for the dark energy, but rather to test the accuracy of our approximation against a variety of different possibilities.

The PNGB model [48], has a potential given by

$$
V(\phi)=\rho_{\phi 0}+\varepsilon M^{4}[1-\cos (\phi / f)],
$$

where $M$ and $f$ are constants. (For recent discussions of the PNGB model in the context of dark energy, see, e.g., Refs. [49, 50, 51] and references therein). Other models with a local potential minimum include the Gaussian potential,

$$
V(\phi)=\rho_{\phi 0}+\varepsilon M^{4}\left[1-e^{-\phi^{2} / \sigma^{2}}\right],
$$

and the quadratic potential

$$
V(\phi)=\rho_{\phi 0}+\varepsilon V_{2} \phi^{2} .
$$

where $\sigma$ and $V_{2}$ are constants. We set initial conditions deep within the matter-dominated regime. The value of the potential at the extremum, $\rho_{\phi 0}$, is chosen to be equal to the energy of the cosmological constant. The initial velocity of the field is taken to be zero.

As discussed above, our formalism applies to models for which (2) is satisfied, but (3) is not. The initial value of the field $\phi_{i}$ determines the accuracy of the first slowroll condition (2) at the initial time. To push our formalism to its limits, we choose $\phi_{i}$ to a value for which

$$
\left[\frac{1}{V} \frac{d V}{d \phi}\right]_{a \rightarrow 0}^{2} \lesssim O[1]
$$

For smaller $\phi_{i}$ of course, the agreement is better.

We have examined three types of curvature of the potential at the extremum, characterized by $K$. The "flat" regime is represented by $0 \leq K^{2}<1$, where $w$ evolves in a slow monotic manner, and $K^{2}=0$ represents a limiting case of this behavior. The "curved" regime is represented by $-\infty<K^{2}<0$ in which $w$ eventually oscillates. In this regime, we have considered the cases of $K^{2}=-10$ and $K^{2}=-20$ for each model.

In Figs. (2-77), the evolution of $w$ from (23) (for $K^{2}=$ 0 ) or (24) (for $K^{2}<0$ ) is shown in comparison to the exact evolution for the three different models, and the different $K$ - regimes described above. The agreement, in all three cases, between our analytic approximation and the exact numerical evolution is excellent, with errors $\delta w \lesssim 0.01$ in all cases. This result indicates that our expressions for $w(a)$ for our three cases of interest can be considered "generic" expressions that apply to a wide range of possible quintessence and phantom models.

Finally, in Figs. (8 [10), we use our analytic approximation (Eqs. 23 24) to construct a $\chi^{2}$ likelihood plot for $w_{0}$ and $\Omega_{\phi 0}$ for the three choices of $K^{2}$ using the recent Type Ia Supernovae standard candle data (ESSENCE+SNLS+HST from [6]). We have exploited

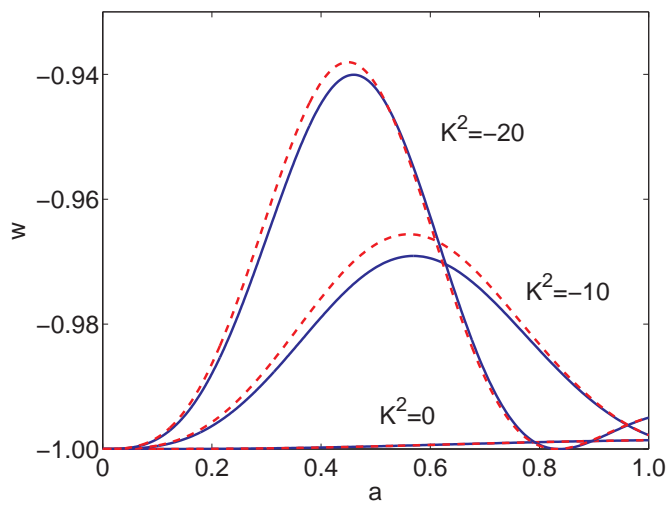

FIG. 2: The evolution of $w$ for quintessence in a quadratic potential for three different values of $K^{2}$. The solid blue curves indicate the exact evolution and the red dashed curves indicate the analytic prediction.

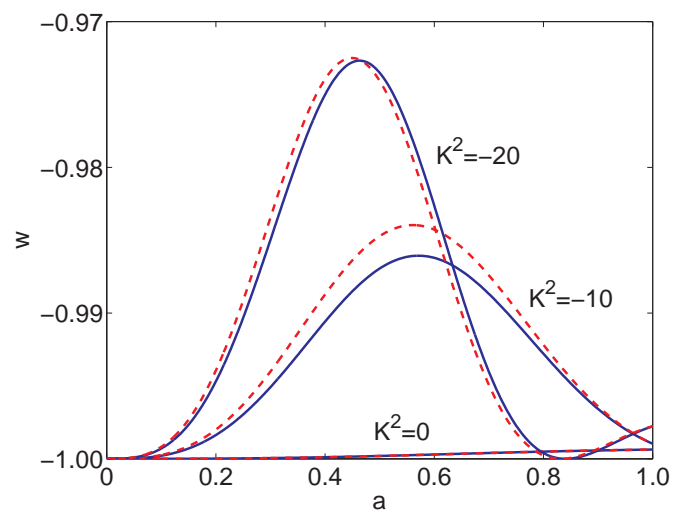

FIG. 3: The evolution of $w$ for quintessence in a PNGB potential for three different values of $K^{2}$. The solid blue curves indicate the exact evolution and the red dashed curves indicate the analytic prediction.

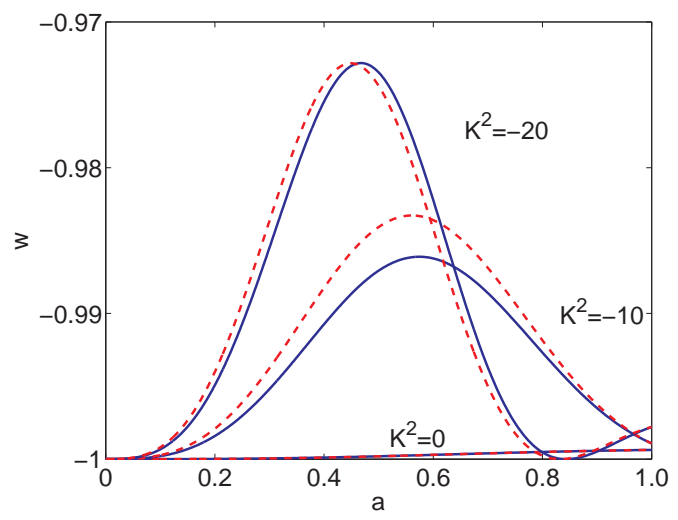

FIG. 4: The evolution of $w$ for quintessence in a Gaussian potential for three different values of $K^{2}$. The solid blue curves indicate the exact evolution and the red dashed curves indicate the analytic prediction. 


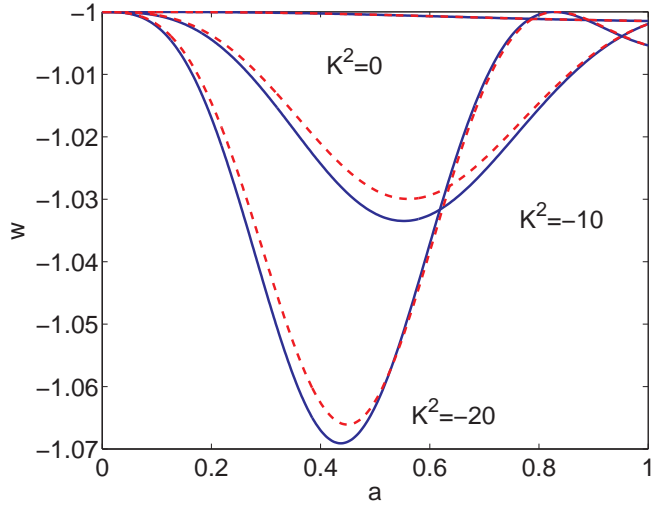

FIG. 5: The evolution of $w$ for a phantom in a quadratic potential for three different values of $K^{2}$. The solid blue curves indicate the exact evolution and the red dashed curves indicate the analytic prediction.

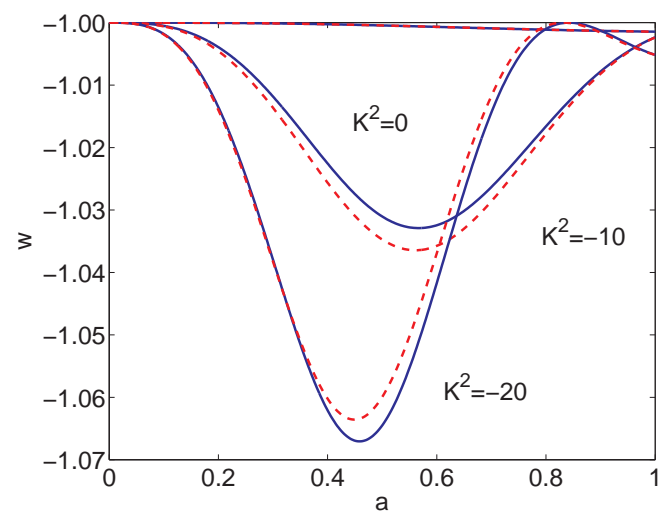

FIG. 6: The evolution of $w$ for a phantom in a PNGB potential for three different values of $K^{2}$. The solid blue curves indicate the exact evolution and the red dashed curves indicate the analytic prediction.

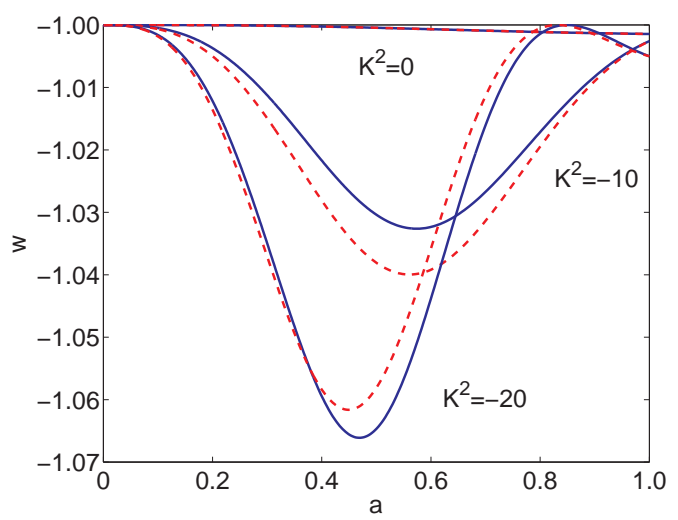

FIG. 7: The evolution of $w$ for a phantom in a Gaussian potential for three different values of $K^{2}$. The solid blue curves indicate the exact evolution and the red dashed curves indicate the analytic prediction.

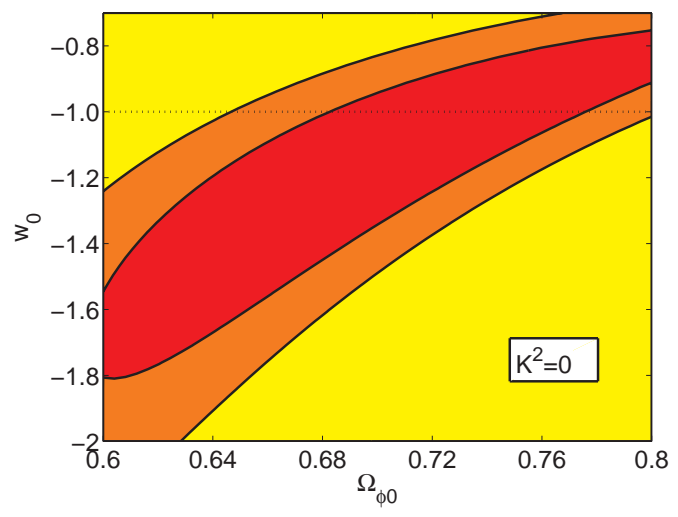

FIG. 8: Likelihood plot from SNIa data for the parameters $w_{0}$ and $\Omega_{\phi 0}$, for quintessence and phantom models with generic behavior described by Eq. (23), with $K^{2}=0$, where $K$ is the function of the curvature of the potential at its extremum given in Eq. (19). The yellow (light) region is excluded at the $2 \sigma$ level, and the darker (orange) region is excluded at the $1 \sigma$ level. Red (darkest) region is not excluded at either confidence level.

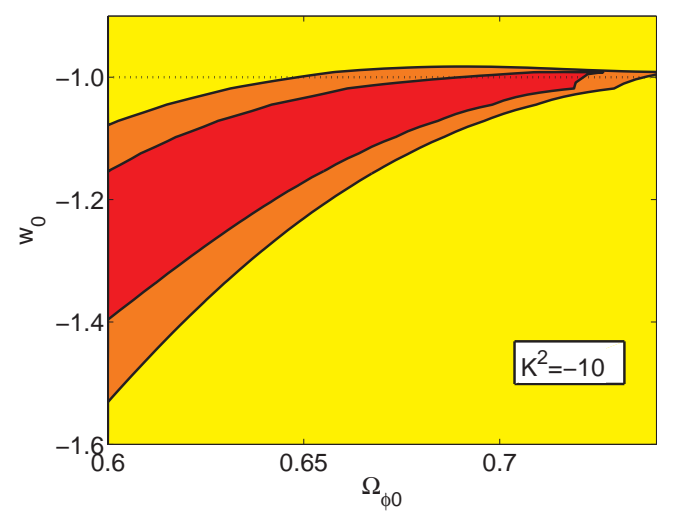

FIG. 9: Likelihood plot from SNIa data for the parameters $w_{0}$ and $\Omega_{\phi 0}$, for quintessence and phantom models with generic behavior described by Eq. (24), with $K^{2}=-10$, where $K$ is the function of the curvature of the potential at its extremum given in Eq. (19). The yellow (light) region is excluded at the $2 \sigma$ level, and the darker (orange) region is excluded at the $1 \sigma$ level. Red (darkest) region is not excluded at either confidence level.

the fact that our expressions for $w(a)$ for quintessence and phantom models have the same functional form, allowing us to produce a likelihood plot that is continuous across $w_{0}=-1$. However, it is important to note that in these figures, the dashed line at $w_{0}=-1$ divides two distinct models. Clearly, these models are not ruled out by current supernova data. 


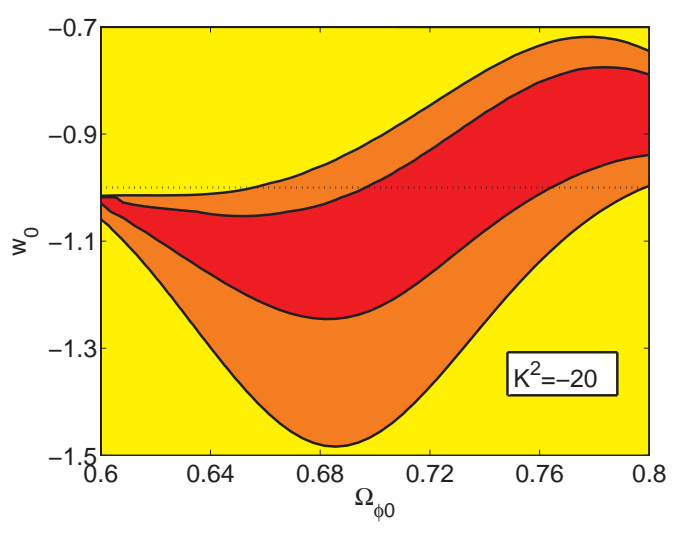

FIG. 10: Likelihood plot from SNIa data for the parameters $w_{0}$ and $\Omega_{\phi 0}$, for quintessence and phantom models with generic behavior described by Eq. (24), with $K^{2}=-20$, where $K$ is the function of the curvature of the potential at its extremum given in Eq. (19). The yellow (light) region is excluded at the $2 \sigma$ level, and the darker (orange) region is excluded at the $1 \sigma$ level. Red (darkest) region is not excluded at either confidence level.

\section{CONCLUSIONS}

Using techniques previously applied in [39, 40, 41], we have derived a general expression for the evolution of $w$, which is valid for a wide class of quintessence and phantom dark energy models in which the field is rolling close to a stable local potential extremum (i.e., a minimum for the quintessence and a maximum for the phantom case, respectively). Such models provide a mechanism to produce a value of $w$ that is close to -1 . We have tested our expression against the (numerically determined) exact evolution for three different representative models and in each case it replicated the exact evolution studied with an accuracy greater than 99\%. A comparison between our generic approximation and the observational data indicates that these models are allowed by SNIa data for a variety of values of the potential curvature parameter $K$ defined in equation (19).

Finally, we note that the case considered here, in which $V^{\prime \prime}>0$ for quintessence (and $V^{\prime \prime}<0$ for phantom models) leads to a much richer set of behaviors than the previously-examined case of $V^{\prime \prime}<0$ for quintessence (and $V^{\prime \prime}>0$ for phantom models). In the case examined here, we see three very different regimes, depending on the sign of $K^{2}$.

\section{Acknowledgments}

We thank T. Chiba for helpful discussions. S.D. and R.J.S. was supported in part by the Department of Energy (DE-FG05-85ER40226).
[1] R. A. Knop et al. [Supernova Cosmology Project Collaboration], Astrophys. J. 598, 102 (2003).

[2] A.G. Riess, et al., Ap.J. 607, 665 (2004).

[3] E.J. Copeland, M. Sami, and S. Tsujikawa, Int. J. Mod. Phys. D 15, 1753 (2006).

[4] M. Kowalski et al., Astrophys. J. 686, 749 (2008) arXiv:0804.4142 [astro-ph]].

[5] W.M. Wood-Vasey, et al., Astrophys. J. 666, 694 (2007).

[6] T.M. Davis, et al., Astrophys. J. 666, 716 (2007).

[7] B. Ratra and P. J. E. Peebles, Phys. Rev. D 37, 3406 (1988).

[8] C. Wetterich, Nucl. Phys. B 302, 668 (1988).

[9] M. S. Turner and M. J. White, Phys. Rev. D 56, 4439 (1997).

[10] R. R. Caldwell, R. Dave and P. J. Steinhardt, Phys. Rev. Lett. 80, 1582 (1998).

[11] A. R. Liddle and R. J. Scherrer, Phys. Rev. D 59, 023509 (1999).

[12] P. J. Steinhardt, L. M. Wang and I. Zlatev, Phys. Rev. D 59, 123504 (1999).

[13] R.R. Caldwell, Phys. Lett. B 545, 23 (2002).

[14] S. M. Carroll, M. Hoffman and M. Trodden, Phys. Rev. D 68, 023509 (2003).

[15] J. M. Cline, S. Jeon and G. D. Moore, Phys. Rev. D 70, 043543 (2004).

[16] R. V. Buniy and S. D. H. Hsu, Phys. Lett. B 632, 543 (2006).
[17] R. V. Buniy, S. D. H. Hsu and B. M. Murray, Phys. Rev. D 74, 063518 (2006).

[18] Z.-K. Guo, Y.-S. Piao, and Y.-Z. Zhang, Phys. Lett. B 594, 247 (2004).

[19] E. Elizalde, S. Nojiri, and S.D Odintsov, Phys. Rev. D 70, 043539 (2004).

[20] S. Nojiri and S.D. Odintsov, Phys. Rev. D 70, 103522 (2004).

[21] J.-G. Hao and X.-Z. Li, Phys. Rev. D 70, 043529 (2004).

[22] I. Y. Aref'eva, A. S. Koshelev and S. Y. Vernov, Theor. Math. Phys. 148, 895 (2006) [Teor. Mat. Fiz. 148, 23 (2006)]

[23] E. N. Saridakis, arXiv:0811.1333 [hep-th].

[24] L. Perivolaropoulos, Phys. Rev. D 71, 063503 (2005).

[25] M. Sami, A. Toporensky, Mod. Phys. Lett. A 19, 1509 (2004).

[26] V. Faraoni, Class. Quant. Grav. 22, 3235 (2005).

[27] T. Chiba, Phys. Rev. D 73, 063501 (2006).

[28] J. Kujat, R.J. Scherrer, and A.A. Sen, Phys. Rev. D 74, 083501 (2006).

[29] M. R. Setare and E. N. Saridakis, Phys. Lett. B 671, 331 (2009).

[30] B. Feng, X. L. Wang and X. M. Zhang, Phys. Lett. B 607, 35 (2005).

[31] Z. K. Guo, et al., Phys. Lett. B 608, 177 (2005).

[32] B. Feng, M. Li, Y.-S. Piao and X. Zhang, Phys. Lett. B 634, 101 (2006). 
[33] Y. f. Cai, H. Li, Y. S. Piao and X. m. Zhang, Phys. Lett. B 646, 141 (2007).

[34] M. R. Setare and E. N. Saridakis, Phys. Lett. B 668, 177 (2008).

[35] M. R. Setare and E. N. Saridakis, JCAP 0809, 026 (2008);

[36] R. J. Scherrer and A. A. Sen, Phys. Rev. D 77, 083515 (2008).

[37] R. J. Scherrer and A. A. Sen, Phys. Rev. D 78, 067303 (2008).

[38] M. R. Setare and E. N. Saridakis, Phys. Rev. D 79, 043005 (2009).

[39] S. Dutta and R. J. Scherrer, Phys. Rev. D 78, 123525 (2008).

[40] S. Dutta and R. J. Scherrer, arXiv:0902.1004 [astroph.CO].

[41] T. Chiba, arXiv:0902.4037 [astro-ph.CO].
[42] V. Sahni and L. Wang, Phys. Rev. D 62, 103517 (2000).

[43] S. D. H. Hsu, Phys. Lett. B 567, 9 (2003) arXiv:astro-ph/0305096.

[44] E. Masso, F. Rota, and G. Zsembinszki, Phys. Rev. D 72, 084007 (2005).

[45] J.-A. Gu, arXiv:0711.3606

[46] S. Dutta and R. J. Scherrer, Phys. Rev. D 78, 083512 (2008) arXiv:0805.0763 [astro-ph]].

[47] M. C. Johnson and M. Kamionkowski, Phys. Rev. D 78, 063010 (2008).

[48] J.A. Frieman, C.T. Hill, A. Stebbins, and I. Waga, Phys. Rev. Lett. 75, 2077 (1995).

[49] K. Dutta and L. Sorbo, Phys. Rev. D 75, 063514 (2007).

[50] A. Abrahamse, A. Albrecht, M. Barnard, and B. Bozek, Phys. Rev. D 77, 103503 (2008).

[51] R. de Putter and E.V. Linder, arXiv:0808.0189 